Discussion Paper No. 949

\title{
HIERARCHICAL EXPERIMENTATION
}

Chia-Hui Chen

Junichiro Ishida

October 2015

The Institute of Social and Economic Research Osaka University

6-1 Mihogaoka, Ibaraki, Osaka 567-0047, Japan 


\title{
Hierarchical Experimentation
}

\author{
Chia-Hui Chen* and Junichiro Ishida ${ }^{\dagger}$
}

October 7, 2015

\begin{abstract}
We consider a bandit problem faced by a team of two heterogeneous players. The team is hierarchical in that one (the principal) retains the exclusive right to terminate the project while the other (the agent) focuses strictly on implementing the project assigned to him. As a key departure, we assume that the principal may be privately informed about the project quality. In contrast to the existing literature, the belief in our model is generally non-monotonic: while each failure makes the agent less confident in the project, the uninformed principal drops out gradually over time, which partially restores his confidence. We derive explicit solutions for the agent's effort and the principal's exit decisions, which allow us to obtain a full characterization of the equilibrium. We also discuss the role of effort monitoring in this context and suggest a new rationale for delegation.
\end{abstract}

JEL Classification Number: D82, D83

Keywords: bandit problems, experimentation, hierarchy, role specialization, war of attrition, delegation.

\footnotetext{
*Institute of Economic Research, Kyoto University. Email: chchen@kier.kyoto-u.ac.jp

${ }^{\dagger}$ Institute of Social and Economic Research, Osaka University. Email: jishida@iser.osaka-u.ac.jp
} 


\section{Introduction}

Suppose that a manager and a worker jointly experiment with a project of unknown quality. The team is organized hierarchically with clear role specialization where the manager retains the exclusive right to decide when to terminate the project while the worker focuses strictly on running the project assigned to him. There is asymmetry of information between the two parties: the manager has access to her own sources of information and is hence better informed about the prospect of the project. The worker knows that the manager has superior information, but does not know precisely what she knows, in particular how much confidence she has in the project.

The fact that the manager may be privately informed about the project quality totally alters the strategic nature of the problem, because her termination (or continuation) decisions partially reveal her private information. Conducting experiments in this environment then yields two conflicting effects. On one hand, as time lapses without any sign of success, skepticism about the project looms large and naturally diminishes the worker's motivation. On the other hand, given that the project survives, the fact that the principal has not terminated the project reflects her confidence, which partially restores the worker's motivation. The latter possibility is the novel aspect of this situation which sets it apart from the standard setup of experimentation. How should the worker allocate his effort over time in such an environment? At what point should the manager give up and terminate the project? And most importantly, how do these decisions dynamically interact with each other?

Examples of this situation abound in reality. Searching for new ideas and innovations by trial and error is obviously an essential aspect of economic activities. Truly valuable innovations are very rare to come by, however, because they require many different qualities such as vision and leadership to set the right course of action and relentless effort to work through adversities. Since it is very difficult, or nearly impossible, for a single individual to provide all those qualities, this whole process often involves decisions of many individuals at various stages. In most firm organizations, for instance, key strategic decisions are made by higher-level officers because they tend to possess superior information and judgement. In many cases, though, resources required for production, such as time and expertise, reside with lower-level workers, and various operational decisions must be delegated to them. In principle, a similar pattern of role specialization emerges more or less when a group of individuals with different degrees of experience jointly engage in a project of exploratory nature, examples of which range from a fledgling entrepreneur working with a venture capitalist on a business startup to a graduate student collaborating with a professor on a research project. 
Given the ever increasing complexity of economic activities, some extent of role specialization, as dictated by comparative advantages, is clearly indispensable. The vast majority of production units are in fact organized in a hierarchial manner with clear role specialization, as exemplified by the separation of planning and execution - a hallmark of modern corporations. As is often the case, however, the presence of information asymmetry hampers collaborations of this kind. When valuable information is dispersed within a team, informed parties may attempt to hide undesirable information while uninformed ones may haggle to squeeze it, thereby limiting the benefit of role specialization and the team's ability to experiment with new ideas.

To analyze this dynamic conflict and its consequences, we consider a bandit problem faced by a team of two players, referred to as the principal and the agent, who are endowed with different sets of productive resources. The basic setup builds on a standard (two-armed) bandit problem where the team owns a project to experiment with, which is either good or bad and can succeed only if it is good. We extend this canonical setup in two important ways. First, the team we consider here is hierarchical in the sense described above: at each instance, the agent chooses how much effort to supply to the project; conditional on no success having occurred, the principal decides whether to terminate the project. Second, we assume that the principal is potentially better informed about the project quality than the agent. To be more precise, she knows that the project is good for sure with some probability (the informed type), whereas she has no more information than the agent with the remaining probability (the uninformed type). The principal's type, either informed or not, is her private information which the agent cannot observe directly. The game ends either when the agent attains a success or when the principal decides to terminate the project.

We obtain several findings within this framework. First, we derive explicit solutions for the agent's effort level and the principal's exit rate, which allow us to obtain a full characterization of the equilibrium. We in particular show that despite rather complicated dynamic interactions, the equilibrium of our model takes a very simple form consisting of at most three phases:

1. Experimentation phase: The agent supplies maximum effort, and the uniformed type never drops out. The game in this phase is like a standard two-armed bandit problem with one safe arm where the belief monotonically decreases over time.

2. Screening phase: The agent lowers the effort level to screen out the uniformed type who starts dropping out gradually over time. The game in this phase is like the war of attrition with implementation delays on the equilibrium path. The screening phase is 
the defining feature of our model which would never arise under complete information.

3. No-uncertainty phase: Only the informed type survives and all the uncertainty regarding the project quality is resolved. The agent supplies maximum effort indefinitely until he attains a success.

Each of the first two phases may or may not exist while the final no-uncertainty phase always exists, meaning that the equilibrium of our model is characterized by the lengths of the first two phases.

A key determining factor of the nature of the equilibrium - in particular the lengths of the first two phases - is the initial prior belief. Note that the belief of our model is defined in two dimensions: the belief that the principal is informed, and the belief that the project is good, conditional on the principal being uninformed. Interestingly, although an increase in either dimension enhances the agent's prior confidence in the project, each comes with a totally different, and in fact opposite, welfare implication. In general, an increase in the former shortens the screening phase and is welfare-improving whereas an increase in the latter prolongs it and is welfare-reducing. ${ }^{1}$ As a practical interpretation, one may argue that more trust in the principal's evaluation ability or "vision" raises efficiency but a more optimistic outlook on the project harms welfare.

The equilibrium is often, though not always, inefficient largely due to the the screening phase which necessarily entails an implementation delay. ${ }^{2}$ During the screening phase, the agent keeps his effort inefficiently low to screen out the uniformed type who responds by dropping out gradually over time. This draws clear contrast to the efficient allocation under which the agent always supplies maximum effort to the project until the belief falls below some threshold, and the uninformed type terminates once and for all at the threshold belief.

We also discuss the role of effort monitoring in this dynamic context. When effort is observable, an additional increment of effort directly lowers the uninformed type's belief. As this forces the uninformed type to drop out at a faster pace, the agent has an extra incentive to raise effort. An interesting point is that although one may think that this can only benefit the agent, this conjecture does not always hold true in this setup: the agent's expected payoff can either increase or decrease with observable effort. To see why, it is important to note that the uninformed type's continuation payoff depends on the current belief as well as the expected future effort sequence. On one hand, when the agent supplies more effort, the belief

\footnotetext{
${ }^{1}$ To be more precise, we obtain this result when the initial project quality, i.e., the initial prior probability of the project being good conditional on the agent's information set, is fixed.

${ }^{2}$ The efficient benchmark here is the case with symmetric information in which the agent knows the principal's type.
} 
will decline at a faster pace, which accelerates the uninformed type's learning and bring forward the timing of project termination. On the other hand, there is also a countervailing effect: knowing that the agent has a stronger incentive to exert effort, the principal expects a higher continuation payoff and is less willing to terminate the project. The agent thus faces a time-inconsistent objective. When the latter aspect dominates the former, better effort monitoring actually reduces the agent's expected payoff.

Finally, our analysis illuminates that prolonged implementation delays can occur as a means to extract the principal's private information. One way to solve this dynamic conflict is therefore to delegate the termination right entirely to the agent, so that he would have no incentive to wait for the principal's action. We show that this form of delegation indeed improves the overall efficiency of the team under some conditions even though this necessarily entails the cost of losing the principal's potentially valuable information. The result suggests that hiving no information at all as a team can be better than having some imperfect information when the information is sparsely distributed.

Related literature: There are now increasingly many works, often called strategic experimentation, where a group of individuals, rather than a single individual, face bandit problems with informational externalities among them. ${ }^{3}$ A prototype of this strand of literature, such as Bolton and Harris (1999), Keller et al. (2005), and Bonatti and Hörner (2011), considers the case where a team of homogeneous players collaborate on a common project (perfectly correlated payoffs). ${ }^{4}$ In contrast, we consider a team of heterogeneous individuals who are endowed with different sets of resources and therefore are in charge of different sets of tasks.

With few exceptions, most recent works of strategic experimentation assume that only one type can succeed, and hence one success can resolve all the uncertainty. Due to this structure, which may be called the "breakthrough type," the game effectively ends as soon as a success is achieved. ${ }^{5}$ While breakthrough-type models are highly tractable and provide crucial insights that would otherwise be more obscure, one inevitable consequence of this specification is that any player's belief is by design monotonically decreasing over time; as such, those models exhibit no "encouragement effect." 6 This draws clear contrast to our

\footnotetext{
${ }^{3}$ An early application of the bandit problem is Rothschild (1974). See Bergemann and Välimäki (2008) for a succinct survey.

${ }^{4}$ Other examples of strategic experimentation include Bergemann and Välimäki (2000), Decamps and Mariotti (2004), Strulovici (2010) and Klein and Rady (2011) among others. Chen and Ishida (2014) analyze a "tenure-clock problem" in which the principal retains the right to terminate the project (or the employment relationship). There, the situation is opposite in that the agent privately observes his own type which the principal attempts to uncover through experimentation.

${ }^{5}$ Early models of strategic experimentation, such as Bolton and Harris (1999) and Bergemann and Välimäki (2000), consider a setting where the belief follows a Wiener process. However, due to the technical complexity of the Brownian model, many recent models of strategic experimentation adopt the breakthrough specification.

${ }^{6}$ The encouragement effect is one of the analytical focuses of Bolton and Harris (1999) whereby the presence
} 
setting in which, even though it also retains the breakthrough-type structure, the agent's belief in general follows a non-monotonic path, as the continuation of the project serves as an encouraging news for the agent.

The current model also has an inherent connection to the war of attrition under incomplete information; in fact, it can be seen as a hybrid of experimentation and war-of-attrition models. Our work bears certain resemblance to reputational models of bargaining in which a player may "irrationally" stick to his own demand and never concede (Kambe, 1999; Abreu and Gul, 2000; Wolitzky, 2012). Given that a player may be irrationally stubborn, even a player who is not has an incentive to behave as if he is stubborn, so as to convince the other party that he will never give in. While our setup differs from theirs in many ways, ${ }^{7}$ a similar economic principle applies to the screening phase of our game: the informed type is like the stubborn type who never concedes, although here rationally, and the uninformed type acts as if she is informed until the last minute.

Finally, there is a large body of literature which discusses whether and under what conditions delegation (or decentralization) dominates more centralized decision-making. ${ }^{8}$ Many works on this topic depict delegation as a means to incorporate local information, with emphasis on the tradeoff between better information and loss of control (e.g., Aghion and Tirole, 1997; Dessein, 2002; Acemoglu et al., 2007). ${ }^{9}$ Our work is also related to this strand of literature in that it displays a virtue of delegation. Note, however, that the information structure of our setting is totally different from theirs as it is the principal who possesses superior information, and centralized control is adopted in the first place precisely because of this informational advantage. As such, the way delegation works in this context in fact goes in the opposite direction: here, delegation is a commitment device to refrain from the principal's information, thereby eliminating asymmetry of information that could otherwise be the cause of inefficient implementation delays.

of other players, and the possibility that they will bring in useful information in future, provide an additional incentive for current experimentation.

${ }^{7}$ The most notable difference is that the uninformed type is unsure of the project quality, and must hence gather information about it over time. This learning process is in general absent in those reputational models of bargaining.

${ }^{8} \mathrm{~A}$ large body of literature on this topic centers around the Revelation Principle which implies that centralized contracting cannot be dominated by hierarchial contracting. Given this fact, attention has been paid to cases where the Revelation Principle fails to hold, e.g., due to costly communication/information processing or collusion among agents. See Mookherjee (2006) for an excellent survey from this mechanism design perspective.

${ }^{9}$ Aghion and Tirole (1997) analyze the impact of the allocation of authority on the incentive to acquire information with particular focus on the distinction between real and formal authority. Dessein (2002) considers cheap talk communication in organizations, and shows that delegation emerges as the optimal choice as long as the incentive conflict is not too large. Acemoglu et al. (2007) examine a situation where a firm must decide whether to adopt a new technology and argue that the firm delegates control to the manager when the value of local information is high. 


\section{Model}

Environment: We consider a problem faced by a team consisting of a principal (female) and an agent (male). The team is hierarchical and characterized by the separation of planning and execution. To be more precise, the principal is conferred authority to terminate the project at any time. The agent is endowed with $\lambda$ units of perfectly divisible resource at his disposal and faces a canonical two-armed bandit problem in which he allocates a fraction of his resource to implement the project.

Information: The team owns a project of unknown quality. The expected return of the project depends on its quality $\theta \in\{0,1\}$ which is either $\operatorname{good}(\theta=1)$ or bad $(\theta=0)$. The principal is either informed or uninformed about the project type: with probability $q_{0} \in(0,1)$, she is informed and knows that the project is good for sure; with probability $1-q_{0}$, she is uninformed and believes that the project is good with prior probability $p_{0} \in(0,1) .{ }^{10}$ The agent has no information of his own, aside from $q_{0}$ and $p_{0}$ that are common knowledge.

Project implementation: The outcome of the project is either a success or a failure at each instance, where the agent can achieve a success or a "breakthrough" only if the project is good. At each instance, the agent chooses how much of his resource to allocate to the project, conditional on the continuation of the game. Let $a_{t} \in[0, \lambda]$ denote the amount of the resource allocated at time $t$, which we refer to as the effort level for expositional clarity throughout the analysis. If the agent chooses $a_{t}=a$ for $t \in[t, t+d t)$, he achieves a success with probability $a_{t} \theta d t .{ }^{11}$ The outcome is publicly observable, and the game ends immediately when the agent achieves a success. For most part, we assume that the principal cannot directly observe $a_{t}$.

Project termination: At each instance, conditional on no success having occurred, the principal chooses either to terminate the project and take the outside option or to continue the project (for expositional purposes, we often say that the principal "drops out" when she terminates the project). The principal terminates the project when she believes that its continuation payoff is not high enough to justify further experimentation. The decision to terminate the project is irreversible, and the game ends once the principal chooses to exercise this option. The value of the outside option for the principal is $b$ which summarizes all the opportunity costs of implementing the project. The agent, on the other hand, receives a payoff of zero once the project is terminated.

\footnotetext{
${ }^{10}$ The project type can alternatively be interpreted as reflecting the agent's productivity or innate ability. Under this interpretation, the principal is assumed to possess superior information about how productive the agent is, as often emphasized in social psychology as well as in works such as Benabou and Tirole (2003), Ishida (2006) and Swank and Visser (2007).

${ }^{11}$ This means that if the effort level is constant over time, the arrival of a success has an exponential distribution. This specification, which is now common in the literature, is due to Keller et al. (2005).
} 
Payoffs: A success yields a non-transferrable benefit, normalized to unity, to each party. The instantaneous payoff for the principal in period $t$ is given by

$$
u_{t}^{P}=y_{t}
$$

where $y_{t}=1$ if the worker attains a success and $y_{t}=0$ otherwise. The instantaneous payoff for the agent is given by

$$
u_{t}^{A}=y_{t}-c a_{t}
$$

where $c \in(0,1)$ measures the effort cost (or the opportunity cost of implementing the project). A crucial aspect of the model is that the effort cost is borne entirely by the agent, so that the interests of the two players are not perfectly aligned. Both players are risk neutral and maximize the discounted sum of payoffs, with a common discount rate given by $r$.

\section{Analysis}

\subsection{Equilibrium concept}

Throughout the analysis, we adopt the concept of perfect Bayesian equilibrium (PBE). As is well known, a potential problem with PBE is that it often admits a plethora of equilibria, including those that do not appear intuitively reasonable. This is also the case in the current setup because the following set of strategies may constitute a PBE: for some $t^{\prime}$, the agent chooses $a_{t}=0$ for all $t \geq t^{\prime}$, and both types of the principal choose to terminate the project altogether at time $t^{\prime}{ }^{12}$ We argue that this is less reasonable as an equilibrium outcome, given that the informed type always has a strictly larger incentive to continue the project than the uninformed type: under this incentive structure, one could argue that if anyone drops out, then the uninformed type must do so no later than the informed type. In fact, this is precisely the spirit of the D1 criterion proposed by Banks and Sobel (1987). Throughout the analysis, therefore, we restrict our attention to those perfect Bayesian equilibria that survive the D1 criterion, which we refer to as non-trivial for the sake of exposition.

Definition 1 A non-trivial equilibrium is a PBE satisfying the D1 criterion.

It is intuitively clear that when $b$ is large relative to the value of a success (which is normalized at one), there only exists an equilibrium in which the uninformed type terminates the project immediately at time 0 , resolving any information asymmetry at the outset. Since

\footnotetext{
${ }^{12}$ Given that $a_{t}=0$ for $t \geq t^{\prime}$, the continuation payoff is zero for both types. This set of strategies then constitutes a $\mathrm{PBE}$ if the agent holds a very pessimistic belief off the equilibrium path and continues to choose $a_{t}=0$ even after a deviation.
} 
this equilibrium is obviously uninteresting for the purpose of our study, we further restrict our attention to the case where $b$ is relatively small, so that the uninformed type stays in the game for a positive duration of time.

Assumption $1 p_{0}>\frac{B r}{\lambda}$ where $B:=\frac{b}{1-b}$.

\subsection{Belief updating}

The informed type knows that the project is good, and as such, her belief stays constant over time regardless of the history of the game. In contrast, both the agent and the uninformed type gradually update their beliefs about the project quality from the observed sequence of outcomes. Due to the initial information asymmetry, however, they hold different expectations regarding the eventual likelihood of a success. Formally, the belief of this game is two-dimensional and denoted by $\left(p_{t}, q_{t}\right)$, where $p_{t}$ denotes the belief that the project is good conditional on the principal being uninformed while $q_{t}$ denotes the belief that the principal is informed. Purely for expositional purposes, we refer to $p_{t}$ as the uninformed type's belief and to $q_{t}$ as the agent's belief although, more accurately, $p_{t}$ also constitutes part of the agent's belief. As noted above, the initial prior $\left(p_{0}, q_{0}\right)$ is a key parameter of the model which characterizes the underlying production environment.

For a given effort sequence $\left\{a_{s}\right\}_{s \in[0, t]}$ up to time $t$, the uninformed type's belief is computed as

$$
p_{t}=\frac{p_{0} e^{-\int_{0}^{t} a_{s} d s}}{1-p_{0}+p_{0} e^{-\int_{0}^{t} a_{s} d s}},
$$

provided that the game still continues. As is well known, the law of motion for the belief is given by

$$
\dot{p}_{t}=-a_{t} p_{t}\left(1-p_{t}\right)
$$

The uninformed type's belief $p_{t}$ is relatively simple and well-behaved as it only depends on $a_{t}$. With $a_{t}$ bounded between 0 and $\lambda$, one can see that it is always continuous and weakly decreasing in $t$.

It is, on the other hand, more complicated to compute the agent's belief, as it depends on the rate at which the principal terminates the project as well as his own effort choice. In principle, both types of the principal can terminate the project when the continuation payoff is sufficiently low compared to the value of the outside option. As it turns out, though, we can show that the informed type never drops out under Assumption 1 (see Lemma 1 below). Except for some point masses at which the uniformed type terminates the project 
with positive probability (with an infinite density), the principal's termination strategy can be represented by an exit rate $x_{t}$, which is the rate at which the uninformed type terminates the project.

Let $q_{t}$ denote the agent's belief that the principal is informed at time $t$. For clarity, we sometimes refer to $p_{t}^{A}:=q_{t}+\left(1-q_{t}\right) p_{t}$ as the project quality which is the probability of the project being good conditional on the set of information available to the agent. Given that $x_{s} \in[0, \infty)$ for $s \in\left[t^{\prime}, t\right.$ ) (i.e., no discontinuity in $q_{t}$ ), the agent's belief is computed as

$$
q_{t}=\frac{q_{t^{\prime}} e^{-\int_{t^{\prime}}^{t} a_{s} d s}}{\left(1-q_{t^{\prime}}\right) e^{-\int_{t^{\prime}}^{t}\left(p_{s} a_{s}+x_{s}\right) d s}+q_{t^{\prime}} e^{-\int_{t^{\prime}}^{t} a_{s} d s} .} .
$$

As above, the law of motion for the belief is

$$
\dot{q}_{t}=\left(x_{t}-\left(1-p_{t}\right) a_{t}\right) q_{t}\left(1-q_{t}\right)
$$

Unlike the uninformed type's belief that can only go down, the agent's belief can go up and down, depending on the exit rate. In fact, it may not even be continuous, for the uninformed type may terminate the project with positive probability. Throughout the analysis, we assume that $q_{t}$ is left-continuous and sometimes denote the right-hand limit by $\tilde{q}_{t}:=\lim _{s \downarrow t} q_{s}$ to indicate " the belief immediately after time $t . " 13$

\subsection{Some useful properties}

We begin our analysis by establishing two useful properties that must hold in any non-trivial equilibrium. The first property is that the informed type never terminates the project as we claimed above. That is, if it is not optimal for the informed type to terminate the project at time 0 , it is never optimal to do so at any subsequent time. This property is highly convenient as it allows us to focus on the uninformed type's problem and substantially simplifies our analysis.

Lemma 1 Under Assumption 1, the informed type never terminates the project in any nontrivial equilibrium.

Proof: To see this, note first that the expected instantaneous payoff for the informed type is $a_{t}$ whereas that for the uninformed one is $p_{t} a_{t}$ for any given $a_{t}$. It is thus clear that the incentive to continue the project is always strictly larger for the informed type. This, along with the D1 criterion, means that the informed type terminates the project only if $q_{t}=1$

\footnotetext{
${ }^{13}$ In an interval with $x_{t}<\infty$, the belief is continuous and $\tilde{q}_{t}=q_{t}$. If the uninformed type terminates the project with probability $\pi$ at some time $t$, then $\tilde{q}_{t}=\frac{q_{t}}{(1-\pi)\left(1-q_{t}\right)+q_{t}}$.
} 
(after the uniformed type completely drops out), at which point all the uncertainty regarding the project quality is resolved.

Given this fact, the lemma is almost self-evident. We first show that the agent chooses the maximum effort level if there exists some $T$ such that $q_{t}=1$ for $t>T$. With no uncertainty, the current effort choice has no future implication, so that the problem is essentially a static one where the agent only needs to maximize the instantaneous payoff $(1-c) a_{t}$. Given that $c<1$, it is optimal for the agent to choose $a_{t}=\lambda$ for $t>T$.

Since the informed type's belief is constant, her continuation payoff is also constant when the agent always chooses the maximum effort level. The optimal choice for the informed type is hence either to stop now or never. Given that the agent exerts maximum effort, the informed type chooses never to stop if

$$
\int_{0}^{\infty} \lambda e^{-(\lambda+r) t} d t=\frac{\lambda}{\lambda+r}>b \Leftrightarrow \lambda>B r
$$

which holds under Assumption 1.

The second useful property is that the probability that the uniformed type continues the project must reach zero in some finite time, meaning that the uncertainty regarding the project type will eventually be resolved at some point.

Lemma 2 There exists some finite $T$ such that $q_{t}=1$ and $a_{t}=\lambda$ fort $t>T$ in any non-trivial equilibrium.

Proof: Suppose otherwise. For this to be the case, the uninformed type's belief $p_{t}$ must remain high enough to continue the project. This means that for any $\varepsilon>0$, there must exist some $t_{\varepsilon}$ such that $a_{t}<\varepsilon$ for all $t>t_{\varepsilon}$. However, this is not compatible with the equilibrium condition because when $\varepsilon$ is arbitrarily close to zero, the continuation payoff is also arbitrarily close to zero for any $t>t_{\varepsilon}$, prompting the uniformed type to terminate the project. The fact that $a_{t}=\lambda$ when $q_{t}=1$ follows from the proof of Lemma 1.

\subsection{The agent's problem}

As we have seen, the uninformed type completely drops out by time $T$ and $q_{t}=1$ for all $t>T$. The agent chooses $a_{t}=\lambda$ for $t>T$, knowing that the project is good for sure. The continuation payoff for the agent from that point on is then given by

$$
V_{T}=\int_{0}^{\infty} \lambda(1-c) e^{-(\lambda+r) t} d t=\frac{\lambda}{\lambda+r}(1-c) .
$$


Given $p_{t}$ and $q_{t}$, the agent's value function is

$$
\begin{aligned}
V_{t}\left(p_{t}, q_{t} ; T\right)= & q_{t}\left(\int_{t}^{T}(1-c) a_{s} e^{-\int_{t}^{s}\left(a_{u}+r\right) d u} d s+e^{-\int_{t}^{T}\left(a_{s}+r\right) d s} V_{T}\right) \\
& +\left(1-q_{t}\right) \int_{t}^{T}\left(p_{s}-c\right) a_{s} e^{-\int_{t}^{s}\left(p_{u} a_{u}+x_{u}+r\right) d u} d s \\
= & q_{t}\left(\int_{t}^{T}(1-c) a_{s} e^{-\int_{t}^{s}\left(a_{u}+r\right) d u} d s+e^{-\int_{t}^{T}\left(a_{u}+r\right) d u} V_{T}\right) \\
& +\left(1-q_{t}\right)\left(p_{t} \int_{t}^{T}(1-c) a_{s} e^{-\int_{t}^{s}\left(a_{u}+x_{u}+r\right) d u} d s-\left(1-p_{t}\right) \int_{t}^{T} c a_{s} e^{-\int_{t}^{s}\left(x_{u}+r\right) d u} d s\right),
\end{aligned}
$$

for $t \leq T .{ }^{14}$ By the Principle of Optimality, the value function must satisfy

$$
V_{t}(p, q)=\max _{a \in[0, \lambda]}\left\{\left(p^{A}-c\right) a d t+e^{-\left(p^{A} a+(1-q) x+r\right) d t} E\left[V_{t+d t}(p+d p, q+d q) \mid a\right]\right\},
$$

where $p^{A}:=q+(1-q) p$. Since

$$
E\left[V_{t+d t}(p+d p, q+d q) \mid a\right]=V_{t}(p, q)-\left(p a(1-p) \frac{\partial V_{t}}{\partial p}-(p a+x-a) q(1-q) \frac{\partial V_{t}}{\partial q}-\dot{V}_{t}\right) d t,
$$

by letting $d t \rightarrow 0$ and rearranging, we obtain the Bellman equation:

$$
V_{t}(p, q)=\frac{1}{r+(1-q) x}\left(q x(1-q) \frac{\partial V_{t}}{\partial q}+\dot{V}_{t}+\max _{a \in[0, \lambda]} \Phi_{t}(p, q) a\right),
$$

where

$$
\Phi_{t}(p, q):=p^{A}-c-p(1-p) \frac{\partial V_{t}}{\partial p}-(1-p) q(1-q) \frac{\partial V_{t}}{\partial q}-p^{A} V_{t}(p, q) .
$$

The Bellman equation implies that the optimal effort is governed by $\Phi_{t}$ where

$$
a_{t} \begin{cases}=\lambda & \text { if } \Phi_{t}\left(p_{t}, q_{t}\right)>0 \\ \in[0, \lambda] & \text { if } \Phi_{t}\left(p_{t}, q_{t}\right)=0 \\ =0 & \text { if } 0>\Phi_{t}\left(p_{t}, q_{t}\right) .\end{cases}
$$

Since

$$
\begin{aligned}
\frac{\partial V_{t}}{\partial p}= & (1-q)\left(\int_{t}^{T}(1-c) a_{s} e^{-\int_{t}^{s}\left(a_{u}+x_{u}+r\right) d u} d s+\int_{t}^{T} c a_{s} e^{-\int_{t}^{s}\left(x_{u}+r\right) d u} d s\right) \\
\frac{\partial V_{t}}{\partial q}= & \left(\int_{t}^{T}(1-c) a_{s} e^{-\int_{t}^{s}\left(a_{u}+r\right) d u} d s+e^{-\int_{t}^{T}\left(a_{u}+r\right) d u} V_{T}\right) \\
& -\left(p \int_{t}^{T}(1-c) a_{s} e^{-\int_{t}^{s}\left(a_{u}+x_{u}+r\right) d u} d s-(1-p) \int_{t}^{T} c a_{s} e^{-\int_{t}^{s}\left(x_{u}+r\right) d u} d s\right)
\end{aligned}
$$

\footnotetext{
${ }^{14}$ More precisely, this expression holds when the exit rate is finite. However, the argument remains essentially the same even if there is a point mass in the exit rate. Suppose that the uninformed type terminates the project with probability $\pi$ at some time $\tau \in(t, T)$. In this case, the integral is improper, and the probability that the uninformed type stays in the game throughout this interval is given by $(1-\pi) e^{-\lim _{z \uparrow \tau} \int_{t}^{z} x_{s} d s-\lim _{z \downarrow \tau} \int_{z}^{T} x_{s} d s}$. With abuse of notation, we let $\pi:=1-e^{-\int_{\tau}^{\tau} x_{\tau} d s}$ and denote $e^{-\int_{t}^{T} x_{s} d s}=(1-\pi) e^{-\lim _{z \uparrow \tau} \int_{t}^{z} x_{s} d s-\lim _{z \downarrow \tau} \int_{z}^{T} x_{s} d s}$ throughout the analysis.
} 
by the envelope theorem, ${ }^{15}$ we have

$$
\begin{aligned}
\Phi_{t}(p, q)= & p^{A}-c-q\left(\int_{t}^{T}(1-c) a_{s} e^{-\int_{t}^{s}\left(a_{u}+r\right) d u} d s+e^{-\int_{t}^{T}\left(a_{u}+r\right) d u} V_{T}\right) \\
& -p(1-q) \int_{t}^{T}(1-c) a_{s} e^{-\int_{t}^{s}\left(a_{u}+x_{u}+r\right) d u} d s,
\end{aligned}
$$

which can be used to characterize the agent's effort choice.

\subsection{The uninformed type's problem}

The uninformed type decides when to terminate the project taking the current belief $p_{t}$ as given. Clearly, it is optimal for the uninformed type to continue the project when the continuation payoff is strictly above $b$ while it is never optimal when it is strictly below $b$. When the continuation payoff equals $b$, the uninformed type may drop out at some positive rate $x_{t}>0$. To be more precise, for a time interval in which $x_{t} \in(0, \infty)$, the uninformed type must be indifferent between terminating and continuing, which implies the following condition to be satisfied.

Lemma 3 If there exists an open interval I such that $x_{t} \in(0, \infty)$ for $t \in I$, then $p_{t} a_{t}=B r$ for $t \in I$.

PROOF: The proof follows the standard argument from the war of attrition. If the principal terminates the project at time $t$, she earns $b$; if she terminates the project at the next instance, she earns $p_{t} a_{t} d t+b\left(1-r d t-p_{t} a_{t} d t\right)$. The uninformed principal is thus indifferent and randomizes between terminating and continuing for any $t \in I$ only if $p_{t} a_{t}=B r$.

Given that $a_{t} \leq \lambda$, Lemma 3 implies that $\underline{p}:=\frac{B r}{\lambda}$ is the lowerbound of $p_{t}$, below which the uniformed principal has no incentive to continue the project any further. Moreover, because $a_{t}=\lambda$ for $t>T$ and $p_{t}$ is continuous, the belief must reach this lowerbound at time $T$, i.e., $p_{t}>p_{T}=\underline{p}$ for $t<T$.

\section{Equilibrium characterization}

We have seen that in any non-trivial equilibrium, there exists some finite $T$ such that $a_{t}=\lambda$ and $q_{t}=1$ for $t>T$ with $p_{T}=\underline{p}$. Before time $T$, the uninformed type remains with some positive probability and may gradually drop out as time passes. What, then, happens just before the game reaches time $T$ ? The fact that the uninformed type persists until time $T$, but

\footnotetext{
${ }^{15}$ See Milgrom and Segal (2002)
} 
not beyond, implies that there must be a small number $\varepsilon$ such that $a_{t}>0$ for $t \in(T-\varepsilon, T]$. Along with the lowerbound of $p_{t}$, this suggests that there must also exist some lowerbound for $q_{t}$, so as to satisfy the agent's incentive compatibility constraint.

Lemma $4 q_{T} \geq \underline{q}:=\frac{c-\underline{p}}{\frac{r}{\lambda+r}(1-c)+(c-\underline{p})}>\frac{c-\underline{p}}{1-\underline{p}}$.

Proof: See Appendix.

Now suppose that there exists some $\tau<T$ such that $a_{t} \in(0, \lambda)$ for $t \in(\tau, T)$. We can then show that during this interval, if it exists, the uninformed type must also drop out at some positive (yet finite) rate to keep the agent indifferent. These indifferent conditions imply some additional conditions that the belief pair $\left(p_{t}, q_{t}\right)$ must satisfy, which can be used to characterize the equilibrium of the model.

Lemma 5 Suppose that there exists some $\tau<T$ such that $a_{t} \in(0, \lambda)$ for $t \in(\tau, T)$. Then, $x_{t} \in(0, \infty)$ for $t \in(\tau, T)$. During this phase, $a_{t}$ is strictly increasing in $t$ with $\lim _{t \uparrow T} a_{t}=\lambda$ whereas $x_{t}, p_{t}$ and $q_{t}$ are all strictly decreasing with $q_{T}=\underline{q}$. Moreover,

$$
q_{t}+\left(1-q_{t}\right) p_{t}>c>p_{t}
$$

for $t \in(\tau, T)$.

Proof: See Appendix.

With the preceding results, we can now make the following statement which provides a characterization of the equilibrium.

Proposition 1 For any given $p_{0}$ and $q_{0}$, there exists $\tau \in[0, T]$ such that

$$
a_{t}\left\{\begin{array} { l l } 
{ = \lambda } & { \text { for } t \in [ 0 , \tau ) , } \\
{ \in ( 0 , \lambda ) } & { \text { for } t \in ( \tau , T ) , }
\end{array} \text { and } x _ { t } \left\{\begin{array}{ll}
=0 & \text { for } t \in[0, \tau), \\
\in(0, \infty) & \text { for } t \in(\tau, T) .
\end{array}\right.\right.
$$

If $T>\tau$, given $p_{\tau}$ and $\tilde{q}_{\tau}$, the effort level and the exit rate follow

$$
\begin{gathered}
a_{t}=\frac{B r e^{-B r(t-\tau)}}{e^{-B r(t-\tau)}-\left(1-p_{\tau}\right)}, \\
x_{t}=-r+\frac{-1+\frac{c}{\left(1-p_{\tau}\right) e^{B r(t-\tau)}-(1-c)}}{-\frac{1}{r}+\frac{c}{r(1-c)} \sum_{n=1}^{\infty} \frac{1}{1+n B}\left(\frac{1-c}{1-p_{\tau}}\right)^{n} e^{-n B r(t-\tau)}+\Omega_{\tau} e^{r(t-\tau)}},
\end{gathered}
$$

for $t \in(\tau, T)$ where

$$
\Omega_{\tau}:=\frac{1}{r}\left(1+\frac{\left(1-\tilde{q}_{\tau}\right) p_{\tau}}{\tilde{q}_{\tau}(1-c)}-\frac{c}{1-c} \sum_{n=1}^{\infty} \frac{1}{1+n B}\left(\frac{1-c}{1-p_{\tau}}\right)^{n}\right) .
$$


Proof: See Appendix.

The proposition states that any non-trivial equilibrium consists of at most three phases and is completely characterized by a pair $(\tau, T)$. For expositional clarity, we label them as follows:

Experimentation phase $t \in[0, \tau]$ : The agent allocates all of his resource to implement the project, and the uniformed type never drops out. The game in this phase is like a standard two-armed bandit problem with one safe arm where a single agent allocates his resource between the two arms.

Screening phase $t \in(\tau, T]$ : The agent controls his effort to screen out the uniformed principal who starts dropping out gradually over time. The game in this phase is like a war of attrition where the agent attempts to screen out the uniformed type by controlling effort, and the uninformed type drops out gradually over time.

No-uncertainty phase $t \in(T, \infty)$ : Only the informed principal survives with no uncertainty regarding the project quality.

The final no-uncertainty phase always exists in any non-trivial equilibrium whereas each of the other two may not under certain conditions. As we will discuss below, key determining factors of $(\tau, T)$ are the prior belief $\left(p_{0}, q_{0}\right)$, and the upper bound of effort $\lambda$. In what follows, we thus think of $\tau$ and $T$ as functions of $\left(p_{0}, q_{0}, \lambda\right)$ and examine how they depend on those parameters.

To construct an equilibrium of this model, it is convenient to summarize several properties that must be satisfied by the belief pair $\left(p_{t}, q_{t}\right)$.

1. $p_{t}$ strictly decreases over time and reaches $\underline{p}$ at time $T$ [Lemmas 3 and 5].

2. $q_{t}$ strictly decreases for $t \in[0, \tau]$ and $(\tau, T]$ with $q_{T} \geq \underline{q}$ (and a possible jump at time $\tau)$ [Lemma 5].

3. If $T>\tau, q_{T}=\underline{q}$ and $q_{t}+\left(1-q_{t}\right) p_{t}>c>p_{t}$ for $t \in(\tau, T)$ [Lemma 5].

4. At time $T, q_{t}$ jumps up to one and stays there for all $t>T$ [Lemma 2].

Note that given (4), the uniformed type's belief at time $T$ is obtained as

$$
p_{T}=\frac{p_{0} e^{-\left(\lambda \tau+\int_{\tau}^{T} a_{s} d s\right)}}{1-p_{0}+p_{0} e^{-\left(\lambda \tau+\int_{\tau}^{T} a_{s} d s\right)}},
$$


which must equal $\underline{p}$ by property 1 . Simple computation shows that

$$
\lambda \tau+\int_{\tau}^{T} a_{s} d s=A\left(p_{0}\right):=\ln \left[\frac{(1-\underline{p}) p_{0}}{p\left(1-p_{0}\right)}\right]
$$

regardless of $(\tau, T)$, meaning that the total amount of effort over $[0, T]$ is fixed at $A\left(p_{0}\right)$ in any non-trivial equilibrium. This fact then implies that since $a_{t}<\lambda$ for $t \in(\tau, T)$, an increase in $\tau$ must be accompanied by a decrease in $T$ and hence $T-\tau$.

Given the initial prior belief $\left(p_{0}, q_{0}\right)$ and the equilibrium strategies, $\tau$ and $T$ are determined so as to satisfy those four properties, with discrete jumps of $q_{t}$ at time $T$ and also possibly at time $\tau$. Note that from property 3 , the screening phase cannot exist if $\underline{p} \geq c$, as the uninformed type's belief would not reach $c$ before time $T$. In this case, therefore, the equilibrium takes a very simple form: the agent chooses $a_{t}=\lambda$ for all $t$, and the uninformed type drops out once and for all at time $T$ when the agent's belief reaches $\underline{p}$.

If $c>\underline{p}$, on the other hand, the equilibrium may involve the screening phase. To actually construct an equilibrium, we first suppose that $x_{t}<\infty$ for $t \in[0, T)$, so that the agent's belief is continuous everywhere (up to time $T$ ). Define $\ell:=T-\tau$ as the length of the screening phase. The uniformed type's belief in this phase can be derived from (4), (5) and the terminal condition $p_{T}=\frac{B r}{\lambda}$. Since $c>p_{t}$ must hold in this phase, there is an upperbound of $\ell$, denoted as $\bar{\ell}$, such that $p_{T-\bar{\ell}}=c$ if $c>\frac{B r}{\lambda}$. Let

$$
L\left(p_{0}\right):=\left\{(\tau, T) \mid \text { given } p_{0}, \tau, T \in(\tau, \tau+\bar{\ell}), \text { and } x_{\tau}<\infty, p_{T}=\underline{p}\right\}
$$

be the set of $(\tau, T)$ such that $p_{T}=\frac{B r}{\lambda}$ for a given initial prior $p_{0}$, assuming no discontinuity of $q_{t}$ at $t=\tau$. Then, for each $(\tau, T) \in L\left(p_{0}\right)$, there is a correspondent $q_{0}$ such that $q_{T}=\underline{q}$. Given this, we can define

$$
Q\left(p_{0}\right):=\left\{q_{0} \mid \text { given } p_{0} \text { and } x_{\tau}<\infty, q_{T}=\underline{q} \text { for }(\tau, T) \in L\left(p_{0}\right)\right\}
$$

which is connected.

Proposition 2 If $c>\underline{p}$, then for any $p_{0}$, there exists a nonempty interval $Q\left(p_{0}\right)$, defined by (7), such that

1. for $q_{0} \in Q\left(p_{0}\right), \tau \geq 0$ and $T-\tau>0$;

2. for $q_{0} \geq \sup \left\{Q\left(p_{0}\right)\right\}, \tau>0$ and $T-\tau=0$;

3. for $q_{0} \leq \inf \left\{Q\left(p_{0}\right)\right\}, \tau>0$ and $T-\tau=\bar{\ell}$ if $p_{0}>c$, whereas $\tau=0$ and $T-\tau<\bar{\ell}$ if $c \geq p_{0}$. 
If $\underline{p} \geq c$, then $\tau>0$ and $T-\tau=0$.

Proof: See Appendix.

Note that the source of preference incongruence in this setup is the effort cost $c$ that must be privately borne by the agent. When $c$ is sufficiently close to zero, the equilibrium ceases to a simple one where the agent simply exerts maximum effort until the project is terminated. In what follows, therefore, we restrict our attention to the case where $c$ is relatively large to ensure that the interests of the two players are sufficiently divergent.

\section{Assumption $2 c>\underline{p}$.}

The efficiency of the equilibrium depends heavily on the length of the screening phase measured by $\ell:=T-\tau$ : as a rule of thumb, the equilibrium becomes less efficient as $\ell$ becomes larger. An important question is hence how $\ell$ changes with the initial prior. The answer to this question is relatively straightforward when $q_{0} \notin Q\left(p_{0}\right)$ : if $q_{0}>\sup \left\{Q\left(p_{0}\right)\right\}$ or $q_{0}<\inf \left\{Q\left(p_{0}\right)\right\}$ with $p_{0}>c$, a small change in the initial prior has no effect on $\ell$; if $q_{0}<\inf \left\{Q\left(p_{0}\right)\right\}$ with $c>p_{0}$, an increase in $p_{0}$ prolongs the screening phase while an increase in $q_{0}$ has no effect. ${ }^{16}$ For $q_{0} \in Q\left(p_{0}\right)$, on the other hand, $\ell$ changes continuously with a change in the initial prior. Even in this case, however, we can obtain very clear-cut results.

Proposition 3 For any given $p_{0}$ and $q_{0} \in Q\left(p_{0}\right)$,
1. $\frac{\partial \tau}{\partial q_{0}}>0, \frac{\partial T}{\partial q_{0}}<0$ and $\frac{\partial \ell}{\partial q_{0}}<0$;
2. $\frac{\partial \tau}{\partial p_{0}}<0, \frac{\partial T}{\partial p_{0}}>0$ and $\frac{\partial \ell}{\partial p_{0}}>0$.

Proof: See Appendix.

Note that both an increase in $p_{0}$ and an increase in $q_{0}$ raise the initial project quality $p_{0}^{A}$. As it turns out, however, they yield totally different, and in fact opposite, efficiency implications. To see this, consider $\left(p_{0}^{\prime}, q_{0}^{\prime}\right)$ and $\left(p_{0}^{\prime \prime}, q_{0}^{\prime \prime}\right), q_{0}^{\prime}>q_{0}^{\prime \prime}$, such that $q_{0}^{\prime} \in Q\left(p_{0}^{\prime}\right)$ and $q_{0}^{\prime}+\left(1-q_{0}^{\prime}\right) p_{0}^{\prime}=q_{0}^{\prime \prime}+\left(1-q_{0}^{\prime \prime}\right) p_{0}^{\prime \prime}$. Proposition 3 then implies that the case with $\left(p_{0}^{\prime}, q_{0}^{\prime}\right)$ entails a shorter screening phase and hence yields a higher joint payoff than the case with $\left(p_{0}^{\prime \prime}, q_{0}^{\prime \prime}\right)$. In other words, with the initial project quality $p_{0}^{A}$ being fixed, an increase in $q_{0}$ is welfare-improving whereas an increase in $p_{0}$ is welfare-reducing.

\footnotetext{
${ }^{16}$ Similarly, when $\underline{p} \geq c$, a small change in the initial prior has no effect on $\ell$.
} 
While the current framework offers a wide of range of interpretations, one possible interpretation is that $p_{0}$ reflects the inherent difficulty of the project at hand while $q_{0}$ reflects the principal's evaluation ability or "vision." According to this view, our results imply that more trust in the principal's vision is welfare-improving (a shorter equilibrium delay) but a more optimistic outlook on the project is welfare-reducing (a longer delay). As such, our analysis lends support to the recent leadership literature that emphasizes vision as an essential aspect of effective leadership (e.g., Bennis and Nanus, 1985; Tichy and Devanna, 1986; Conger and Kanungo, 1998) and complements it by presenting a specific mechanism through which vision can become an asset in organizations. ${ }^{17}$

\section{The role of information in hierarchical experimentation}

The model we have considered thus far has both hidden information and hidden action: on one hand, the agent does not know whether the principal is informed or not; on the other, the principal cannot directly observe the agent's resource allocation. Of course, the former aspect is the major focus of our analysis which sets it apart from the existing literature. The latter is more subtle, but nonetheless gives rise to an argument that is instrumental in illustrating the intricate nature of the problem faced by the agent. In this section, we relax each of them in order to illuminate the role of information in the current setup.

\subsection{The efficient benchmark}

To obtain the (constrained) efficient allocation, we consider the case where the agent has both the information and the productive resource to carry out the project. ${ }^{18}$ More precisely, we assume that the agent now knows whether the principal is informed or not. Aside from this, to keep the situation comparable, the model is exactly the same as above; we in particular continue to assume that the principal retains the right to terminate the project.

With no information asymmetry, the problem is enormously simplified: when the agent is informed and knows that the project is good for sure, he simply chooses $a_{t}=\lambda$ until a success is attained; when he is not informed, the problem is effectively reduced to a standard two-armed bandit problem. To obtain the efficient allocation, therefore, we only need to

\footnotetext{
${ }^{17}$ There are also some economic analyses which illustrate the role of vision in organizations from different perspectives. Rotemberg and Saloner (2000) model vision as a bias which makes the manager favor one project over the other. Hiring a manager with a vision can be beneficial because it reduces uncertainty as to what kind of employee initiatives will be favored later. Van den Steen (2005) also formalizes the notion of vision and shows that a leader with strong beliefs would attract employees with similar beliefs. This sorting effect gives direction to the firm and improves coordination, suggesting a channel through which strong vision can affect the firm's performance.

${ }^{18}$ The subsequent benchmark result is constrained efficient in that the players must take the allocation of the implementation costs, as captured by $b$ and $c$, as given.
} 
consider the case where the agent is not informed about the project quality. Since the game ends once the agent attains a success, the optimal choice for the agent is simply to choose $a_{t}=\lambda$ if $p_{t} \geq c$ and $a_{t}=0$ if $c>p_{t}$, both on and off the equilibrium path.

On the other hand, the principal's termination decision is essentially the same. The principal is indifferent between terminating and continuing when $p_{t} a_{t}=B r$. Given the agent's strategy, the principal terminates the project when the belief reaches $\frac{B r}{\lambda}$ if $\frac{B r}{\lambda} \geq c$. If $c>\frac{B r}{\lambda}$, the agent stop exerting effort at $p_{t}=c$. Since the continuation payoff is zero from that point on, it is optimal for the principal to terminate the project when the belief reaches $c$. This means that the principal terminates the project at $p_{t}=\max \left\{\frac{B r}{\lambda}, c\right\}=c$ under Assumption 2.

The distortion caused by the information asymmetry (and the consequent role specialization) should now be clear. When the project is known to be good, the agent should exert maximum effort indefinitely until he attains a success in the efficient allocation. In contrast, the equilibrium allocation often involves a phase where the agent controls effort in some range $(0, \lambda)$, in order to screen out the uninformed type. As such, the information asymmetry often prolongs the experimentation process even though, in the end, the project will end up with a success with probability one when it happens to be good.

The contrast is even more interesting when the project type is not known ex ante. Again, the efficient allocation involves no phase where the agent chooses an intermediate effort level. The belief $p_{t}$ thus declines no faster in the equilibrium allocation than in the efficient one and again slows down the experimentation process. Moreover, in this case, there is also distortion on termination decisions. Since the uninformed type is pooled with the informed type, the marginal value of experimentation is higher, which induces the principal to procrastinate. In equilibrium, the uninformed type starts dropping out at a positive rate only after the belief falls below $c$ and persists until it reaches $\underline{p}$, suggesting that the project continues for too long compared to the efficient benchmark. ${ }^{19}$

\subsection{Observable effort}

Another crucial aspect of our model is that effort is not directly observable to the principal, so that the agent's deviation from the equilibrium strategy would not affect the uninformed type's belief. Although we believe that the case with unobservable effort is a more realistic description of situations of our interest, it is still helpful to consider, at least as a theoretical benchmark, the case where the principal can directly observe the agent's effort level to see

\footnotetext{
${ }^{19}$ If $\underline{p} \geq c$, the agent chooses $a_{t}=\lambda$ for all $t$, and the uninformed type terminates the project once and for all when the belief reaches $\underline{p}$. The project is hence terminated at the right timing with no implementation delay, so that the allocation coincides with the efficient benchmark.
} 
the role of effort monitoring in this context. In particular, our main focus here is to show through an example that the agent's expected payoff can either increase or decrease with observable effort.

A full characterization of the case with observable effort requires as much work, if not more, and is out of the scope of our analysis. For this reason, we restrict our attention to a particular equilibrium allocation which we call the full-effort equilibrium, i.e., the one in which the agent exerts maximum effort all the time, and the uniformed type drops out once and for all at time $T$. Letting $\underline{q}^{s}:=\frac{c-\underline{p}}{1-\underline{p}}$ and $\nu:=\frac{\underline{p}\left(1-p_{0}\right)}{p_{0}(1-\underline{p})}$, we obtain the following condition for the full-effort equilibrium.

Proposition 4 With observable effort, the full-effort equilibrium exists if

$$
q_{0} \geq \frac{\underline{q}^{s}\left(1-p_{0}+p_{0} \nu\right)}{\left(1-\underline{q}^{s}\right) \nu+\underline{q}^{s}\left(1-p_{0}+p_{0} \nu\right)} .
$$

Proof: See Appendix.

It follows from Proposition 2 that under Assumption 2, the full-effort equilibrium exists if and only if

$$
q_{0} \geq \sup \left\{Q\left(p_{0}\right)\right\}=\frac{\underline{q}\left(1-p_{0}+p_{0} \nu\right)}{(1-\underline{q}) \nu+\underline{q}\left(1-p_{0}+p_{0} \nu\right)},
$$

when effort is not observable. Proposition 4 then suggests that the condition with unobservable effort is a proper subset of the condition with observable effort. This is because, with observable effort, the agent has an additional incentive to work harder, as more effort directly lowers the uninformed type's belief and thereby forces her to drop out at a faster rate.

Since the agent has better control over the uniformed type's behavior, one might think that he is always better off with observable effort. As it turns out, though, this is not always the case in the current setup because of a time-inconsistent objective that the agent faces. To see this, note that the uninformed type's continuation payoff at time $t$ depends on two factors: the current belief $p_{t}$ and the expected effort sequence $\left\{a_{s}\right\}_{s=t}^{\infty}$. The agent can thus screen out the uninformed type either by exerting high effort (which lowers $p_{t}$ ) or by exerting low effort (which diminishes the continuation payoff). From the agent's point of view, the latter is a more thrifty way to induce the principal's information. When it is more effective, the agent would actually be better off if he could commit to a low-effort sequence, although he would always have an incentive to deviate and exert higher effort in equilibrium.

Intuitively, with observable effort, the uninformed type starts dropping out later (a higher $\tau$ ) due to higher continuation payoffs, but the belief $p_{t}$ reaches the lowerbound $\underline{p}$ earlier (a 
lower $T$ ). The benefit of effort observability for the agent is hence that it takes shorter to reach the no-uncertainty phase where the expected payoff is maximized, whereas the cost is that the uninformed type is more willing to stay in the game. Below, we show that the agent's expected payoff can indeed either increase or decrease with observable effort because of this tradeoff.

Proposition 5 Let $q_{0}=\frac{q^{s}\left(1-p_{0}+p_{0} \nu\right)}{\left(1-\underline{q}^{s}\right) \nu+\underline{q}^{s}\left(1-p_{0}+p_{0} \nu\right)}$, so that the agent chooses $a_{t}=\lambda$ for all $t$ with observable effort, but $a_{t} \in(0, \lambda)$ for some $t$ with unobservable effort.

(i) Fix $B r=K$ for some $K \in(0, \lambda)$. There exists $\varepsilon>0$ such that for $r<\varepsilon$ and $B=\frac{K}{r}$, the agent receives a higher payoff with unobservable effort than with observable effort.

(ii) There exists $\lambda^{*}>0$ such that for $\lambda>\lambda^{*}$, the agent receives a higher payoff with observable effort than with unobservable effort.

Proof: See Appendix.

The uninformed type's incentive to continue the project is determined by $\underline{p}=\frac{B r}{\lambda}$, where a better outside option (a higher $b$ ) implies a higher opportunity cost of continuing the project: in a sense, $\underline{p}$ measures how patiently the principal can wait for a success by forgoing the outside option. Fixing $\underline{p}$, a decrease in $r$ means that the agent becomes more patient, compared to the uninformed type, and is willing to go through a prolonged screening phase: in fact, as $r \rightarrow 0$, the agent faces almost no cost from implementation delays, so that $a_{t} \rightarrow 0$ for $t \in(\tau, T)$ to screen out the uninformed type with unobservable effort. With observable effort, however, the agent cannot do this, as he has a stronger current incentive to exert higher effort at each instance. In other words, the agent obtains a higher expected payoff with unobservable effort because the fact that effort cannot be observed by the principal works as a commitment device to keep his effort low enough.

In contrast, effort observability increases the agent's expected payoff when the upperbound of the effort level is sufficiently high. As can be seen from (4) and (5), $\lambda$ has no impact on $a_{t}$ and $x_{t}$ for $t \in(\tau, T)$. The difference is that the maximum instantaneous payoff is high for a given belief $p_{t}$, and the uninformed type is therefore more willing to wait for a success even when the belief is relatively low. Since the equilibrium necessarily involves the screening phase with unobservable effort by assumption, the agent must now go through a prolonged screening phase, which diminishes his expected payoff. 


\subsection{Delegation and the value of the principal's information}

The inefficiency of the equilibrium arises largely from the screening phase in which the agent lowers effort to extract the principal's private information. As is evident, one potential remedy for this is to take the principal entirely out of the game and delegate the termination right to the agent even though it necessarily entails a cost of losing the principal's (potentially useful) information. Here, we explore whether this type of delegation can ever improve the productive efficiency of the team as measured by the joint payoff of the principal and the agent.

The optimal strategy under delegation is fairly straightforward. The agent exerts maximum effort until the belief $p_{t}^{A}$ reaches $c$ and then terminates the project. The cost of delegation in this context is obvious: with no additional information, the agent must terminate the project once and for all at some point, independently of the project quality. This means that a good project is terminated prematurely with some probability while a bad project on average persists for too long. However, there is also a potential gain from delegation because the agent now knows that he can no longer rely on the principal's information and so always exerts maximum effort as long as he continues the project.

Can this benefit be large enough to more than compensate for the cost? As it turns out, the answer to this question is yes, i.e., the team as a whole can be made better off by giving up the principal's information entirely under some conditions. The argument here runs parallel to part (ii) of Proposition 5: roughly, the benefit dominates the cost when both $\lambda$ and $q_{0}$ are sufficiently large. As $q_{0}$ approaches one, the project is good almost surely, and the risk of having a bad project for too long also diminishes to zero. Even in this case, when $\lambda$ is sufficiently large, the equilibrium without delegation involves some implementation delay (see Lemma 8 in the proof of Proposition 5), leading to the following proposition.

Proposition 6 Let $q_{0}=\frac{q^{s}\left(1-p_{0}+p_{0} \nu\right)}{\left(1-\underline{q}^{s}\right) \nu+\underline{q}^{s}\left(1-p_{0}+p_{0} \nu\right)}$. There exists $\lambda^{* *}$ such that delegation raises the joint payoff for $\lambda>\lambda^{* *}$.

Proof: The argument here is very closely related to part (ii) of Proposition 5, especially Lemma 8. Note that since $\lim _{\lambda \rightarrow \infty} q_{0}=1$, the project is almost surely good as $\lambda$ tends to infinity. This means that the project succeeds almost surely by the time the belief reaches $\underline{p}$. The allocation under delegation thus converges to the efficient one that maximizes the joint payoff. In contrast, it follows from Lemma 8 that the equilibrium without delegation must involve a prolonged screening phase with a probability that is bounded away from zero and hence yield a strictly lower joint payoff than the efficient allocation. 


\section{Conclusion}

This paper provides a model of hierarchical experimentation in which a team of heterogeneous players faces a bandit problem in order to understand how the presence of information asymmetry limits the team's ability to explore new ideas. We derive explicit solutions for the agent's effort choice and the principal's exit rate that are used to obtain a full characterization of the equilibrium. The equilibrium is often, though not always, inefficient, with prolonged delays occurring on the equilibrium path, which constrains the benefit of role specialization. In general, more trust in the principal's vision improves welfare whereas more optimistic outlook on the project lowers it.

\section{References}

Abreu, D. and Gul, F., 2000, Bargaining and Reputation, Econometrica, 68, 85-117.

Acemoglu, D., Aghion, P., Lelarge, C., Van Reenen, J. and Zilibotti, F., 2007, Technology, Information, and the Decentralization of the Firm, Quarterly Journal of Economics, 122, 1759-99.

Aghion, P. and Tirole, J., 1997, Formal and Real Authority in Organization, Journal of Political Economy, 105, 1-29.

Banks, J.S. and Sobel, J., 1987, Equilibrium Selection in Signaling Games, Econometrica, 55, 647-61.

Benabou, R. and Tirole, J., 2003, Intrinsic and Extrinsic Motivation, Review of Economic Studies, 70, 489-520.

Bennis, W.G. and Nanus, B., 1985, Leaders: The Strategies for Taking Charge, New York: Harper and Row.

Bergemann, D. and Välimäki, J., 2000, Experimentation in Markets, Review of Economic Studies, 67, 213-34.

Bergemann, D. and Välimäki, J., 2008, Bandit Problems, in Durlauf, S. and Blume, L., eds, The New Palgrave Dictionary of Economics, 2nd edition, Macmillan Press.

Bolton, P. and Harris, C., 1999, Strategic Experimentation, Econometrica, 67, 349-74.

Bonatti, A. and Hörner, J., 2011, Collaborating, American Economic Review, 101, 632-63.

Chen, C.-H. and Ishida, J., 2015, A Tenure-Clock Problem, ISER Discussion Paper No. 919. 
Conger, J.A. and Kanungo, R.N., 1998, Charismatic Leadership in Organizations, Thousand Oaks, CA: Sage Publications.

Decamps, J.-P. and Mariotti, T., 2004, Investment Timing and Externalities, Journal of Economic Theory, 118, 80-102.

Dessein, W., 2002, Authority and Communication in Organizaitons, Review of Economic Studies, 69, 811-38.

Ishida, J., 2006, Optimal Promotion Policies with the Looking-Glass Effect, Journal of Labor Economics, 24, 857-77.

Kambe, S., 1999, Bargaining with Imperfect Commitment, Games and Economic Behavior, $28,217-37$.

Keller G., Rady, S. and Cripps, M., 2005, Strategic Experimentation with Exponential Bandits, Econometrica, 73, 39-68.

Klein, N.A. and Rady, S., 2011, Negatively Correlated Bandits, Review of Economic Studies, $78,693-732$.

Mookherjee, D., 2006, Decentralization, Hierarchies and Incentives: A Mechanism Design Perspective, Journal of Economic Literature, 44, 367-90.

Milgrom, P. and Segal, I., 2002, Envelope Theorems for Arbitrary Choice Sets, Econometrica, 70, 583-601.

Rotemberg, J.J. and Saloner, G., 2000, Visionaries, Managers, and Strategic Direction, RAND Journal of Economics, 31, 693-716.

Rothschild, M., 1974, A Two-Armed Bandit Theory of Market Pricing, Journal of Economic Theory, 9, 185-202.

Strulovici, B., 2010, Learning While Voting: Determinants of Collective Experimentation, Econometrica, 78, 933-71.

Swank, O.H. and Visser, B., 2007, Motivating through Delegating Tasks or Giving Attention, Journal of Law, Economics, and Organization, 23, 731-42.

Tichy, N.M. and Devanna, M.A., 1986, The Transformational Leader, New York: John Wiley and Sons.

Van den Steen, E., 2005, Organizaitonal Beliefs and Managerial Vision, Journal of Law, Economics, and Organization, 21, 256-83.

Wolitzky, A., 2012, Reputational Bargaining with Minimal Knowledge of Rationality, Econometrica, 80, 2047-87. 


\section{Appendix: Proofs}

Proof of Lemma 4: We first show that there exists a small number $\varepsilon>0$ such that $a_{t}>0$ for $t \in(T-\varepsilon, T)$. Suppose otherwise, i.e., there exists some $t^{\prime}$ such that the agent chooses $a_{t}=0$ for $t \in\left(t^{\prime}, T\right)$. During this time interval, however, the probability of attaining a success is zero, and it is hence strictly better for the uninformed type to terminate the project at time $t^{\prime}$ rather than at time $T$, which is a contradiction.

The fact that the agent exerts some effort when it is sufficiently close to time $T$ implies a certain lowerbound for $q_{t}$. It follows from the Bellman equation that $\lim _{t \uparrow T} a_{t}>0$ only if

$$
\lim _{t \uparrow T} \Phi_{t}\left(p_{t}, q_{t}\right)=\lim _{t \uparrow T}\left(q_{t}+\left(1-q_{t}\right) p_{t}-c-q_{t} V_{T}\right) \geq 0,
$$

which can be written as

$$
q_{T} \geq \frac{c-p_{T}}{\frac{r}{\lambda+r}(1-c)+\left(c-p_{T}\right)}>\frac{c-p_{T}}{1-p_{T}}
$$

Proof of Lemma 5: The agent chooses $a_{t} \in(0, \lambda)$ only if $\Phi_{t}\left(p_{t}, q_{t}\right)=0$. This implies that $\dot{\Phi}_{t}=0$ for $t \in(\tau, T)$. Let

$$
S_{t^{\prime}}^{t}:=q_{t^{\prime}} e^{-\int_{t^{\prime}}^{t} a_{s} d s}+\left(1-q_{t^{\prime}}\right) p_{t^{\prime}} e^{-\int_{t^{\prime}}^{t}\left(a_{s}+x_{s}\right) d s}+\left(1-q_{t^{\prime}}\right)\left(1-p_{t^{\prime}}\right) e^{-\int_{t^{\prime}}^{t} x_{s} d s} .
$$

denote the probability that the game reaches time $t$, conditional on that it has reached time $t^{\prime} \leq t$. Given this, we define a modified version of $\Phi_{t}$ as $\phi_{t}\left(p_{t}, q_{t} ; t^{\prime}\right):=e^{-r\left(t-t^{\prime}\right)} S_{t^{\prime}}^{t} \Phi_{t}\left(p_{t}, q_{t}\right)$, which can be written as

$$
\begin{aligned}
\phi_{t}\left(p_{t}, q_{t} ; t^{\prime}\right)= & \left(q_{t^{\prime}} e^{-\int_{t^{\prime}}^{t}\left(a_{s}+r\right) d s}+p_{t^{\prime}}\left(1-q_{t^{\prime}}\right) e^{-\int_{t^{\prime}}^{t}\left(a_{s}+x_{s}+r\right) d s}\right)(1-c) \\
& -\left(1-q_{t^{\prime}}\right)\left(1-p_{t^{\prime}}\right) e^{-\int_{t^{\prime}}^{t}\left(x_{s}+r\right) d s} c \\
& -q_{t^{\prime}} e^{-\int_{t^{\prime}}^{t}\left(a_{s}+r\right) d s}\left(\int_{t}^{T}(1-c) a_{s} e^{-\int_{t}^{s}\left(a_{u}+r\right) d u} d s+e^{-\int_{t}^{T}\left(a_{u}+r\right) d u} V_{T}\right) \\
& +p_{t^{\prime}}\left(1-q_{t^{\prime}}\right) e^{-\int_{t^{\prime}}^{t}\left(a_{s}+x_{s}+r\right) d s} \int_{t}^{T}(1-c) a_{s} e^{-\int_{t}^{s}\left(a_{u}+x_{u}+r\right) d u} d s .
\end{aligned}
$$

This modified version of $\Phi$ has a desirable property that $\dot{\phi}_{t}\left(p_{t}, q_{t} ; t\right)=0$ if and only if $\dot{\Phi}_{t}\left(p_{t}, q_{t}\right)=0$, provided that $\Phi_{t}\left(p_{t}, q_{t}\right)=0$. Moreover, $\phi_{t}$ and $\Phi_{t}$ always have the same sign. Since the only thing that matters is the sign of $\Phi_{t}$, we use this modified function $\phi$ to characterize the agent's effort choice for analytical convenience. Evaluating at $t^{\prime}=t$, we 
obtain

$$
\begin{aligned}
\dot{\phi}_{t}\left(p_{t}, q_{t} ; t\right)= & -\left(q_{t}\left(a_{t}+r\right)+\left(1-q_{t}\right) p_{t}\left(a_{t}+x_{t}+r\right)\right)(1-c)+\left(1-q_{t}\right)\left(1-p_{t}\right)\left(x_{t}+r\right) c \\
& +q_{t}(1-c) a_{t}+\left(1-q_{t}\right) p_{t}(1-c) a_{t} \\
= & \left(1-q_{t}\right)\left(c-p_{t}\right) x_{t}-\left(q_{t}+\left(1-q_{t}\right) p_{t}-c\right) r .
\end{aligned}
$$

The agent thus chooses $a_{t} \in(0, \lambda)$ for $t \in(\tau, T)$ only if

$$
\left(1-q_{t}\right)\left(c-p_{t}\right) x_{t}=\left(q_{t}+\left(1-q_{t}\right) p_{t}-c\right) r .
$$

Given this, we first show that $x_{t} \in(0, \infty)$ if $a_{t} \in(0, \lambda)$ for $t \in(\tau, T)$. Since $x_{t} \geq 0$ and $q_{t}+\left(1-q_{t}\right) p_{t}>p_{t}$, we must have $q_{t}+\left(1-q_{t}\right) p_{t} \geq c \geq p_{t}$ with at least one of them holding with strict inequality. Suppose that there exists some $t^{\prime} \in(\tau, T)$ such that $c=p_{t^{\prime}}$. Since $p_{t}>p_{t^{\prime}}$ for any $t>t^{\prime}$, we have $q_{t}+\left(1-q_{t}\right) p_{t}>p_{t}>c$ for $t \in\left(\tau, t^{\prime}\right)$, which violates (9). Similarly, suppose that there exists some $t^{\prime} \in(\tau, T)$ such that $q_{t^{\prime}}+\left(1-q_{t^{\prime}}\right) p_{t^{\prime}}=c$ and $x_{t^{\prime}}=0$. Given that $a_{t^{\prime}}>0$ and $x_{t^{\prime}}=0$, both $\dot{p}_{t^{\prime}}$ and $\dot{q}_{t^{\prime}}$ must be negative, meaning that $\dot{p}_{t^{\prime}}^{A}$ is negative as well and (9) can no longer be satisfied. This implies that $q_{t}+\left(1-q_{t}\right) p_{t}>c>p_{t}$, and hence $x_{t} \in(0, \infty)$, for $t \in(\tau, T)$.

To show that $q_{t}$ is decreasing in $t \in(\tau, T)$, we obtain explicit solutions for $a_{t}$ and $x_{t}$. From Lemma 3, we know that $p_{t} a_{t}=B r$ for $t \in(\tau, T)$. This implies that the optimal effort is characterized by the following ordinary differential equation:

$$
\dot{p}_{t} a_{t}+p_{t} \dot{a}_{t}=0 \Leftrightarrow \dot{a}_{t}=-\frac{a_{t}}{p_{t}} \dot{p}_{t}=\frac{a_{t}}{p_{t}} p_{t} a_{t}\left(1-p_{t}\right)=a_{t}\left(a_{t}-B r\right)
$$

Solving this yields

$$
a_{t}=\frac{B r e^{-B r\left(t-t^{\prime}\right)}}{e^{-B r\left(t-t^{\prime}\right)}-\left(1-p_{t^{\prime}}\right)}, p_{t}=1-\left(1-p_{t^{\prime}}\right) e^{B r\left(t-t^{\prime}\right)},
$$

which shows how the effort level evolves over time from an arbitrarily chosen initial point $t^{\prime} \in(\tau, t]$.

As for the exit rate $x_{t}$, note that

$$
x_{t}=\frac{\left(q_{t}+\left(1-q_{t}\right) p_{t}-c\right) r}{\left(1-q_{t}\right)\left(c-p_{t}\right)}=\left(\frac{q_{t}(1-c)}{\left(1-q_{t}\right)\left(c-p_{t}\right)}-1\right) r,
$$

for $t \in(\tau, T)$. With some computation, we obtain

$$
\begin{aligned}
x_{t} & =\left(\frac{(1-c) q_{t^{\prime}}}{\left(c-p_{t}\right)\left(1-q_{t^{\prime}}\right)} e^{\int_{t^{\prime}}^{t}\left(x_{s}-\left(1-p_{s}\right) a_{s}\right) d s}-1\right) r \\
& =\left(\frac{(1-c) q_{t^{\prime}}}{\left(\left(1-p_{t^{\prime}}\right) e^{B r\left(t-t^{\prime}\right)}-(1-c)\right)\left(1-q_{t^{\prime}}\right)} e^{\int_{t^{\prime}}^{t}\left(x_{s}-\frac{B r\left(1-p_{t^{\prime}}\right) e^{B r s}}{1-\left(1-p_{t^{\prime}}\right) e^{B r s}}\right) d s}-1\right) r \\
& =\left(\frac{q_{t^{\prime}}\left(\frac{1}{p_{t^{\prime}}}-\frac{1-p_{t^{\prime}}}{p_{t^{\prime}}} e^{B r\left(t-t^{\prime}\right)}\right)}{\left(1-q_{t^{\prime}}\right)\left(\frac{1-p_{t^{\prime}}}{1-c} e^{B r\left(t-t^{\prime}\right)}-1\right)} e^{B r \int_{t^{\prime}}^{t} x_{s} d s}-1\right) r,
\end{aligned}
$$


for any $t^{\prime} \in(\tau, t]$. By solving (12), we obtain

$$
x_{t}=-r+\frac{-1+\frac{c}{\left(1-p_{t^{\prime}}\right) e^{B r\left(t-t^{\prime}\right)}-(1-c)}}{-\frac{1}{r}+\frac{c}{r(1-c)} \sum_{n=1}^{\infty} \frac{1}{1+n B}\left(\frac{1-c}{1-p_{t^{\prime}}}\right)^{n} e^{-n B r\left(t-t^{\prime}\right)}+\Omega_{t^{\prime}} e^{r\left(t-t^{\prime}\right)}},
$$

where

$$
\Omega_{t^{\prime}}:=\frac{1}{r}\left(1+\frac{\left(1-q_{t^{\prime}}\right) p_{t^{\prime}}}{q_{t^{\prime}}(1-c)}-\frac{c}{1-c} \sum_{n=1}^{\infty} \frac{1}{1+n B}\left(\frac{1-c}{1-p_{t^{\prime}}}\right)^{n}\right) .
$$

With the explicit form of $x_{t}$, we analyze how $q_{t}$ evolves over time. Notice that

$$
e^{\int_{t^{\prime}}^{t} x_{s} d s}=\frac{\left(1-q_{t^{\prime}}\right) p_{t^{\prime}}}{r q_{t^{\prime}}(1-c) \Gamma_{t^{\prime}}^{t}}
$$

where

$$
\Gamma_{t^{\prime}}^{t}:=-\frac{1}{r}+\frac{c}{r(1-c)} \sum_{n=1}^{\infty} \frac{1}{1+n B}\left(\frac{1-c}{1-p_{t^{\prime}}}\right)^{n} e^{-n B r\left(t-t^{\prime}\right)}+\Omega e^{r\left(t-t^{\prime}\right)}
$$

It then follows from this that

$$
\begin{aligned}
\frac{q_{t}}{1-q_{t}} & =\frac{q_{t^{\prime}}}{1-q_{t^{\prime}}}\left(\frac{1}{p_{t^{\prime}}}-\frac{1-p_{t^{\prime}}}{p_{t^{\prime}}} e^{B r\left(t-t^{\prime}\right)}\right) e^{\int_{t^{\prime}}^{t} x_{s} d s} \\
& =\left(\frac{1}{p_{t^{\prime}}}-\frac{1-p_{t^{\prime}}}{p_{t^{\prime}}} e^{B r\left(t-t^{\prime}\right)}\right) \frac{p_{t^{\prime}}}{r(1-c) \Gamma_{t^{\prime}}^{t}} .
\end{aligned}
$$

Note that $q_{t}$ is decreasing in $t$ if $\frac{q_{t}}{1-q_{t}}$ is decreasing. It thus suffices to show that

$$
\frac{d}{d t}\left[\frac{1-\left(1-p_{t^{\prime}}\right) e^{B r\left(t-t^{\prime}\right)}}{\Gamma_{t^{\prime}}^{t}}\right]<0
$$

for any $t^{\prime} \in(\tau, t]$. This can be written as

$$
\left(1-\left(1-p_{t^{\prime}}\right) e^{B r\left(t-t^{\prime}\right)}\right)\left(-r \Gamma_{t^{\prime}}^{t}-1+\frac{c}{\left(1-p_{t^{\prime}}\right) e^{B r\left(t-t^{\prime}\right)}-(1-c)}\right)>-\left(1-p_{t^{\prime}}\right) B r e^{B r\left(t-t^{\prime}\right)} \Gamma_{t^{\prime}}^{t}
$$

Since $\left.\Gamma_{t^{\prime}}^{t}\right|_{t^{\prime}=t}=\frac{\left(1-q_{t}\right) p_{t}}{r q_{t}(1-c)}$, evaluating at $t^{\prime}=t$, this condition becomes

$$
b\left(1-p_{t}\right)\left(1-q_{t}\right)\left(c-p_{t}\right)>p_{t}\left(c-q_{t}-\left(1-q_{t}\right) p_{t}\right),
$$

which holds because $q_{t}+\left(1-q_{t}\right) p_{t}>c$.

As for the effort level, since $a_{t}>0$ implies that $p_{t}$ is strictly decreasing in $t \in(\tau, T), a_{t}$ must increase to satisfy $p_{t} a_{t}=B r$. It then follows from (11) that $x_{t}$ is increasing because both $p_{t}$ and $q_{t}$ are strictly decreasing. Note also that since $\lim _{t \uparrow T} p_{t}=\underline{p}$, we have $\lim _{t \uparrow T} a_{t}=$ 
$\frac{B r}{\underline{p}}=\lambda$. Finally, if there exists some $\tau<T$ such that $a_{t} \in(0, \lambda)$ for $t \in(\tau, T)$, we must have $\Phi_{t}\left(p_{t}, q_{t}\right)=0$ for this range, which means that

$$
q_{T}=\frac{c-p_{T}}{\frac{r}{\lambda+r}(1-c)+\left(c-p_{T}\right)}>\frac{c-p_{T}}{1-p_{T}}
$$

Proof of Proposition 1: To prove the proposition, the following property is crucial.

Lemma 6 Suppose that $p_{t^{\prime}}^{A}=q_{t^{\prime}}+\left(1-q_{t^{\prime}}\right) p_{s}>c$ for some $t^{\prime} \leq T$. If there exists $\varepsilon>0$ such that $a_{t}=\lambda$ for $t \in\left(t^{\prime}-\varepsilon, t^{\prime}\right)$, then $a_{t}=\lambda$ and $x_{t}=0$ for all $t<t^{\prime}$.

Proof: Given that $p_{t}>\frac{B r}{\lambda}$ for $t<T$, if $a_{t}=\lambda$ for $t \in\left(t^{\prime}-\varepsilon, t^{\prime}\right)$, the uninformed type would never terminate the project in $\left[t^{\prime}-\varepsilon, t^{\prime}\right)$. Therefore, $q_{t}$ and $p_{t}$ are strictly decreasing in $t \in\left[t^{\prime}-\varepsilon, t^{\prime}\right)$, and $p_{t}^{A}=q_{t}+\left(1-q_{t}\right) p_{t}>c$ for $t \in\left[t^{\prime}-\varepsilon, t^{\prime}\right)$. Furthermore, given that $a_{t}=\lambda$ for $t \in\left(t^{\prime}-\varepsilon, t^{\prime}\right)$, there also exists $\varepsilon^{\prime}>0$ such that the uninformed type does not terminate the project in $\left(t^{\prime}-\varepsilon-\varepsilon^{\prime}, t^{\prime}-\varepsilon\right]$, regardless of $a_{t}$ during this interval. Given that $x_{t}=0$ in $\left(t^{\prime}-\varepsilon-\varepsilon^{\prime}, t^{\prime}-\varepsilon\right], q_{t}+\left(1-q_{t}\right) p_{t}>c$ for $t \in\left(t^{\prime}-\varepsilon-\varepsilon^{\prime}, t^{\prime}-\varepsilon\right]$. Therefore, $\dot{\phi}_{t}\left(p_{t}, q_{t} ; t\right)$ is negative in $\left(t^{\prime}-\varepsilon-\varepsilon^{\prime}, t^{\prime}-\varepsilon\right]$. This implies $\phi_{t}\left(p_{t}, q_{t} ; t\right)>0 \Leftrightarrow \Phi_{t}\left(p_{t}, q_{t}\right)>0$ for $t \in\left(t^{\prime}-\varepsilon-\varepsilon^{\prime}, t^{\prime}-\varepsilon\right]$ which in turn imply $a_{t}=\lambda$ during this interval. Applying the same argument backward, we can see that $a_{t}=\lambda$ for all $t<t^{\prime}$.

With this result as well as Lemma 5, we are now ready to prove the proposition. We already know that either $a_{t}=\lambda$ or $a_{t} \in(0, \lambda)$ for $t$ sufficiently close to $T$ (see the proof of Lemma 4). It follows from Lemma 6 that if $a_{t}=\lambda$ for $t$ sufficiently close to $T$, then $a_{t}=\lambda$ for all $t<T$. Now suppose that $a_{t} \in(0, \lambda)$ for $t$ sufficiently close to $T$. In this case, we define

$$
\tau:=\inf \left\{\underline{t} \mid a_{t} \in(0, \lambda) \text { for all } t \in(\underline{t}, T)\right\} \text {. }
$$

By Lemma $5, x_{t} \in(0, \infty)$ for $t \in(\tau, T)$, and $\tilde{q}_{\tau}+\left(1-\tilde{q}_{\tau}\right) p_{\tau}>c$.

To prove the proposition, it suffices to show that there exists a small number $\varepsilon>0$ such that $a_{t}=\lambda$ for $t \in(\tau-\varepsilon, \tau]$. By the definition of $\tau$, it is either $a_{\tau}=0$ or $a_{\tau}=\lambda$. We first show that $a_{\tau}=0$ is not feasible. Given that $a_{\tau}=0$, there are two possibilities: (i) $a_{t}=0$ or (ii) $a_{t} \in(0, \lambda)$ for $t$ sufficiently close to $\tau$. If (i) holds, however, the uninformed type should start dropping out before time $\tau$. This is a contradiction because we then must have $a_{t}>0$ to satisfy $p_{t} a_{t}=B r$. If (ii) holds, then $a_{t}$ must be decreasing over time, which contradicts Lemma 5. Now suppose that $a_{t} \in(0, \lambda)$ for $t \in(\tau-\varepsilon, \tau]$ with $a_{\tau}=\lambda$. Along this path, however, $x_{t}=0$ for $t \in(\tau-\varepsilon, \tau]$ because $p_{t} a_{t}>p_{\tau} a_{\tau}=B r$ for $t$ sufficiently close to $\tau$ and 
$p_{t} a_{t}=B r$ cannot be satisfied, but this contradicts Lemma 5 . This shows that $a_{t}=\lambda$ for $t$ sufficiently close to $\tau$. We can then apply Lemma 6 to show that $a_{t}=\lambda$ and $x_{t}=0$ for all $t \in[0, \tau)$.

Explicit solutions for $a_{t}$ and $x_{t}$ are from (10) and (13) in the proof of Lemma 5. Since $p_{t}$ and $q_{t}$ are left-continuous, we obtain the expression in the proposition by replacing $t^{\prime}$ with $\tau$ and $q_{\tau}$ with $\tilde{q}_{\tau}$ (for $q_{t}$ may be discontinuous at time $\tau$ ).

Proof of Proposition 2: We start with the case where $c>\underline{p}$. First, it is clear by definition that there exists a pair $(\tau, T)$ that can satisfy all the equilibrium conditions with no discontinuity at time $\tau$ if $q_{0} \in Q\left(p_{0}\right)$. We can uniquely pin down $\tau$ and $\ell=T-\tau$ from the two boundary conditions, i.e., $q_{T}=\underline{q}$ and $p_{T}=\underline{p}$, as well as (4) and (5). For $q_{0} \geq \sup \left\{Q\left(p_{0}\right)\right\}$, we first establish the following fact.

Lemma 7 For any given $p_{0}$ and $q_{0} \in Q\left(p_{0}\right), \frac{\partial \tau}{\partial q_{0}}>0, \frac{\partial T}{\partial q_{0}}<0$ and $\frac{\partial \ell}{\partial q_{0}}<0$.

Proof: We have already seen that the total amount of effort is fixed at $A\left(p_{0}\right)$ regardless of $(\tau, T)$. In contrast, given (4) and (5), the agent's belief at time $T$ is given by

$$
q_{T}=\frac{q_{0} e^{-\left(\lambda \tau+\int_{\tau}^{T} a_{s} d s\right)}}{\left(1-q_{0}\right) e^{-\left(\int_{0}^{\tau} p_{s} \lambda d s+\int_{\tau}^{T}\left(p_{s} a_{s}+x_{s}\right) d s\right)}+q_{0} e^{-\left(\lambda \tau+\int_{\tau}^{T} a_{s} d s\right)}},
$$

if there is no discontinuity at time $\tau$. The total amount of effort needed to satisfy this condition depends on $(\tau, T)$ because $x_{t}>0$ only for $(\tau, T)$. If $q_{t}$ converges to $\underline{q}$ without any discontinuity, the following condition must be satisfied:

$$
\int_{0}^{\tau}\left(1-p_{s}\right) \lambda d s+\int_{\tau}^{T}\left(\left(1-p_{s}\right) a_{s}-x_{s}\right) d s=B\left(q_{0}\right):=\ln \left[\frac{(1-\underline{q}) q_{0}}{\underline{q}\left(1-q_{0}\right)}\right] .
$$

It follows from (6) and (14) that if there is an exogenous increase in $q_{0}$ while $p_{0}$ is fixed, $\tau$ and $T$ must satisfy

$$
\begin{gathered}
\left(\left(1-p_{\tau}\right)\left(\lambda-a_{\tau}\right)+x_{\tau}\right) \frac{\partial \tau}{\partial q_{0}}+\left(\left(1-p_{T}\right) \lim _{t \uparrow T} a_{t}-\lim _{t \uparrow T} x_{t}\right) \frac{\partial T}{\partial q_{0}}=\frac{d B}{d q_{0}} \\
\left(\lambda-a_{\tau}\right) \frac{\partial \tau}{\partial q_{0}}+\lim _{t \uparrow T} a_{t} \frac{\partial T}{\partial q_{0}}=0 .
\end{gathered}
$$

With some algebra, we obtain

$$
\frac{\partial \tau}{\partial q_{0}}>0 \Leftrightarrow \lim _{t \uparrow T} \frac{x_{t}}{a_{t}}+\frac{x_{\tau}}{a_{T}\left(\lambda-a_{\tau}\right)}>p_{\tau}-p_{T}
$$


Since $x_{t} \geq 0$ and $a_{t} \in[0, \lambda]$, it suffices to show that

$$
\lim _{t \uparrow T} \frac{x_{t}}{a_{t}}>p_{\tau}-p_{T}
$$

From (9) and the fact that $\lim _{t \uparrow T} a_{t}=\lambda$ (from Lemma 5), this condition can be written as

$$
\frac{\left(q_{T}+\left(1-q_{T}\right) p_{T}-c\right) r}{\left(1-q_{T}\right)\left(c-p_{T}\right)}>\lambda\left(p_{\tau}-p_{T}\right) .
$$

Since $c>p_{\tau}$, this holds if

$$
\frac{\left(q_{T}+\left(1-q_{T}\right) p_{T}-c\right) r}{\left(1-q_{T}\right)\left(c-p_{T}\right)}>\lambda\left(c-p_{T}\right)
$$

Substituting $q_{T}=\underline{q}$ and rearranging, we obtain

$$
c-p_{T}+\frac{r}{\lambda+r}(1-c) p_{T}-\left(\frac{r}{\lambda+r}(1-c)+c-p_{T}\right) c>\frac{\lambda}{\lambda+r}(1-c)\left(c-p_{T}\right)^{2},
$$

which is reduced to $1>c-p_{T}$. As this holds by assumption, $\tau$ must increase and $T$ must decrease when there is an increase in $q_{0}$.

Note that Lemma 7 indicates that $\tau$ becomes larger (and $T$ becomes smaller) as $q_{0}$ becomes larger, with $\lim _{q_{0} \rightarrow \sup \left\{Q\left(p_{0}\right)\right\}} \ell=0$, i.e., there is no screening phase when $q_{0}$ is sufficiently large. This suggests that for $q_{0} \geq \sup \left\{Q\left(p_{0}\right)\right\}$, the equilibrium is such that $a_{t}=\lambda$ all the time, and the uninformed type terminates the project once and for all at time $T$ when $p_{t}$ reaches $\underline{p}$.

For $q_{0} \leq \inf \left\{Q\left(p_{0}\right)\right\}$, on the other hand, it should be either (i) $\tau=0$ and $T-\tau<\bar{\ell}$ or (ii) $\tau>0$ and $T-\tau=\bar{\ell}^{20}$ We need to consider two distinct possibilities. If $p_{0}>c$, the agent starts out with maximum effort and the uninformed type drops out with positive probability at time $\tau$ when the belief $p_{t}$ reaches $c$ to satisfy $q_{T}=\underline{q}$. To see that this indeed constitutes an equilibrium, note that since $p_{\tau}=c,(8)$ is negative and $\Phi_{t}\left(p_{t}, q_{t}\right)>0$ for $t \in[0, \tau)$, meaning that $a_{t}=\lambda$ and $x_{t}=0$ in this range. For $t \in(\tau, T]$, the continuation equilibrium is not affected by the jump, so that $a_{t}$ and $x_{t}$ are given by (4) and (5). If $c \geq p_{0}$, on the other hand, the uninformed type is indifferent between terminating and continuing for any $t \in[0, T]$. The uninformed type thus drops out immediately at time 0 so that the revised belief jumps up to satisfy $q_{T}=\underline{q}$, given (4) and (5).

\footnotetext{
${ }^{20}$ Note that there is a sharp discontinuity between the case with $q_{0}=0$ (the symmetric-information case) and the case with an arbitrarily small $q_{0}$. In the latter case, no matter how small $q_{0}$ is to begin with, $q_{t}$ can take any value between zero and one because the uninformed type can drop out with probability arbitrarily close to one. This draws clear contrast to the symmetric-information case where there is zero measure of the informed type, because $q_{0}=0$ regardless of the uninformed type's termination decisions in this case.
} 
If $\underline{p} \geq c, L\left(p_{0}\right)$ is empty for any $p_{0}>\underline{p}$. The screening phase cannot exist, so that $T=\tau$. The agent exerts maximum effort until $p_{t}$ reaches $\underline{p}$, at which point the uniformed type terminates altogether.

Proof of Proposition 3: The first statement is directly from Lemma 7. The second statement can be proved in a similar manner. If there is an exogenous increase in $p_{0}, \tau$ and $T$ must satisfy

$$
\begin{gathered}
\left(\left(1-p_{\tau}\right)\left(\lambda-a_{\tau}\right)+x_{\tau}\right) \frac{\partial \tau}{\partial p_{0}}+\left(\left(1-p_{T}\right) a_{T}-\lim _{t \uparrow T} x_{t}\right) \frac{\partial T}{\partial p_{0}}=0 \\
\left(\lambda-a_{\tau}\right) \frac{\partial \tau}{\partial p_{0}}+a_{T} \frac{\partial T}{\partial p_{0}}=\frac{d A}{d p_{0}}
\end{gathered}
$$

It follows from these that

$$
\frac{\partial \tau}{\partial p_{0}}<0 \Leftrightarrow \frac{\lim _{t \uparrow T} x_{t}}{a_{T}}>p_{\tau}-p_{T}-\frac{x_{\tau}}{a_{T}\left(\lambda-a_{\tau}\right)} .
$$

From Lemma 7, we know that this condition holds. This also implies that $\frac{\partial T}{\partial p_{0}}>0$ to satisfy (6).

Proof of Proposition 4: Given that the agent always exerts maximum effort, the uninformed type has no incentive to terminate the project for $p_{t}>\underline{p}$. Therefore, given $p_{0}$ and $q_{0}$, $q_{t}$ is strictly decreasing over time, and there is a one-to-one mapping between $p_{t}$ and $q_{t}$. As such, we can work with the agent's belief $p_{t}^{A}=q_{t}+\left(1-q_{t}\right) p_{t}$, which is a sufficient statistic as we will see below, instead of keeping track of $p_{t}$ and $q_{t}$ independently. Let $\underline{p}^{A}$ be the correspondent agent's belief when $p_{t}$ reaches $\underline{p}$, and define $T$ such that $p_{T}^{A}=\underline{p}^{A}$. Since the game reaches time $T$ with probability $q_{t} e^{-\lambda(T-t)}$, the agent's value function can be written as

$$
V\left(p^{A}\right)=\lambda\left(\frac{p^{A}(1-c)}{\lambda+r}\left(1-e^{-(\lambda+r)(T-t)}\right)-\frac{\left(1-p^{A}\right) c}{r}\left(1-e^{-r(T-t)}\right)+e^{-(\lambda+r)(T-t)} \frac{q_{t}(1-c)}{\lambda+r}\right) .
$$

Note that the ratio between $p_{t}$ and $q_{t}$ is constant for $t \in[0, T)$, given that $x_{t}=0$ for this interval. This means that

$$
q_{t}=\frac{q_{0}}{p_{0}^{A}} p^{A}
$$

where $p_{t}^{A}:=q_{t}+\left(1-q_{t}\right) p_{t}$. Note also that since $\underline{p}^{A}=\frac{p^{A} e^{-\lambda(T-t)}}{1-p^{A}+p^{A} e^{-\lambda(T-t)}}$, we obtain

$$
e^{-(T-t)}=\left(\frac{p^{A}\left(1-\underline{p}^{A}\right)}{\underline{\underline{p}}^{A}\left(1-p^{A}\right)}\right)^{-\frac{1}{\lambda}}=: \Delta .
$$


It follows from these that

$$
V\left(p^{A}\right)=\lambda\left(\frac{p^{A}(1-c)}{\lambda+r}\left(1-\Delta^{\lambda+r}\right)-\frac{\left(1-p^{A}\right) c}{r}\left(1-\Delta^{r}\right)+\frac{p^{A} q_{0}(1-c)}{p_{0}^{A}(\lambda+r)} \Delta^{\lambda+r}\right) .
$$

Note that the value function depends only on the current belief $p^{A}$, but not on $t$, since now the uninformed type can condition her strategy on the belief $p^{A}$ which is commonly shared even off the equilibrium path. With some computation, we obtain

$$
\frac{d V}{d p^{A}}=\lambda\left(\frac{1-c}{\lambda+r}\left(1-\Delta^{\lambda+r}\right)+\frac{c}{r}\left(1-\Delta^{r}\right)+\frac{q_{0}(1-c)}{p_{0}^{A}(\lambda+r)} \Delta^{\lambda+r}\right)+\frac{(1-c)\left(p_{0}^{A}-q_{0}\right)}{\left(1-p^{A}\right) p_{0}^{A}} \Delta^{\lambda+r}-\frac{c}{p^{A}} \Delta^{r},
$$

The Bellman equation implies that $a_{t}=\lambda$ for $t \in[0, T)$ if

$$
p^{A}-c-p^{A}\left(1-p^{A}\right) \frac{d V}{d p^{A}}-p^{A} V>0 .
$$

A sufficient condition for this is

$$
\psi\left(p^{A}\right):=p^{A}-c-p^{A}\left(\frac{\lambda(1-c)}{\lambda+r}\left(1-\Delta^{\lambda+r}\right)+\left(1-\frac{c}{\underline{p}^{A}}\right) \Delta^{\lambda+r}\right)>0 .
$$

Note that $\psi\left(\underline{p}^{A}\right)=0$ and $\psi(1)=\frac{r}{\lambda+r}(1-c)$. Furthermore,

$$
\psi^{\prime}\left(p^{A}\right)=1-\frac{\lambda}{\lambda+r}(1-c)+\kappa\left(\frac{1}{p^{A}}-1\right)^{1+\frac{r}{\lambda}}\left(1-\frac{\lambda+r}{\lambda\left(1-p^{A}\right)}\right),
$$

where

$$
\kappa:=\left(\frac{\lambda}{\lambda+r}(1-c)-\left(1-\frac{c}{p^{A}}\right)\right)\left(\frac{\underline{p}^{A}}{1-\underline{p}^{A}}\right)^{1+\frac{r}{\lambda}} .
$$

It follows from this that $\psi^{\prime}\left(\underline{p}^{A}\right)=\frac{r}{\lambda\left(1-\underline{p}^{A}\right)}\left(1-\frac{c}{\underline{p}^{A}}\right), \psi^{\prime}(1)=1-\frac{\lambda}{\lambda+r}(1-c)>0$, and

$$
\psi^{\prime \prime}\left(p^{A}\right) \begin{cases}>0 & \text { if } \frac{\lambda}{\lambda+r}(1-c)-\left(1-\frac{c}{\underline{p}^{A}}\right)>0 \\ <0 & \text { if } \frac{\lambda}{\lambda+r}(1-c)-\left(1-\frac{c}{\underline{p}^{A}}\right)<0\end{cases}
$$

When $\underline{p}^{A} \geq c, \psi^{\prime}\left(p^{A}\right)>0$ for all $p^{A}>\underline{p}^{A}$, and hence $\psi\left(p^{A}\right)>0$ for all $p^{A}>\underline{p}^{A}$. This means that the agent chooses $a_{t}=\lambda$ if $\underline{p}^{A} \geq c$.

Given $p_{0}$, to reach $\underline{p}, T$ must satisfy $e^{-\lambda T}=\nu:=\frac{\underline{p}\left(1-p_{0}\right)}{\bar{p}_{0}(1-\underline{p})}$. Given this, $\underline{p}^{A}=q_{T}+\left(1-q_{T}\right) \underline{p} \geq$ $c$, if and only if

$$
q_{0} \geq \frac{\underline{q}^{s}\left(1-p_{0}+p_{0} \nu\right)}{\left(1-\underline{q}^{s}\right) \nu+\underline{q}^{s}\left(1-p_{0}+p_{0} \nu\right)} .
$$


Proof of Proposition 5: Part (i): Let $\left(\tau^{\text {obs }}, T^{\text {obs }}\right)$ denote the equilibrium allocation with observable effort (we continue to denote the allocation with unobservable effort by $(\tau, T)$ ). Given $q_{0}$, with observable effort, the agent always exerts maximum effort $\left(\tau^{\text {obs }}=T^{\text {obs }}\right)$, and the uninformed type drops out once and for all when $p_{t}$ reaches $\frac{K}{\lambda}$ at time $T^{\text {obs }}$. With unobservable effort, by Proposition 2, the uninformed type drops out with strictly positive probability at time $\tau \geq 0$, so that $q_{t}$ jumps up to a level that leads to $\lim _{t \uparrow T} q_{t}=\underline{q}$, which is arbitrarily close to 1 when $r$ approaches 0 . By Lemma $5, q_{t}$ is decreasing, so $q_{t}$ must also be arbitrarily close to 1 after the jump. At time $\tau, p_{\tau}>\frac{K}{\lambda}$, and the agent exerts effort lower than $\lambda$ until $p_{t}$ reaches $\underline{p}$.

We now compare the payoffs when $r$ approaches 0 while fixing $B r=K$. In both cases, the agent chooses $a_{t}=\lambda$ for $t<\tau$, so the agent's expected payoffs are identical up to that point. With unobservable effort, the uninformed type terminates the project almost surely at time $\tau$, so that $q_{\tau}$ jumps up almost to 1 ; with observable effort, the uninformed type waits until $p_{t}$ reaches $\underline{p}=\frac{K}{\lambda}$. Since $p_{t}<c$ for $t>\tau$, the agent incurs a loss if the principal happens to be uninformed, meaning that the agent receives a higher expected payoff with unobservable effort after time $\tau$ and before $p_{t}$ reaches $\underline{p}$ (at $T$ and $T^{\mathrm{obs}}$ ). This is the benefit of unobservable effort, which is strictly positive even when $r$ tends to 0 .

This comes with a cost, however, because the agent exerts effort lower than $\lambda$, so it takes longer for $p_{t}$ to reach $\underline{p}$. Note that given $p_{\tau}$ and the fact that $\underline{p}$ is fixed at $\frac{K}{\lambda}$, the time needed to reach $\underline{p}$ is independent of $r$. As such, the loss which stems from a larger $T$ diminishes to 0 as $r$ tends to 0 . This shows that, for $r$ arbitrarily close to 0 and $B=\frac{K}{r}$, the agent receives a higher payoff with unobservable effort than with observable effort.

Part (ii): The cost and benefit of effort observability are still the same: the benefit is that the game reaches the no-uncertainty phase earlier; the cost is that the uninformed type would not drop out until time $T$. To prove the second part, the following lemma helps.

Lemma 8 Let $q_{0}=\frac{q^{s}\left(1-p_{0}+p_{0} \nu\right)}{\left(1-\underline{q}^{s}\right) \nu+\underline{q}^{s}\left(1-p_{0}+p_{0} \nu\right)}$. With unobservable effort, as $\lambda \rightarrow \infty, e^{-\lambda \tau}$ converges to a positive number, and $\ell:=T-\tau$ goes to $\infty$.

Proof: Note that $\tau$ and $T$ change continuously with $\lambda$. With some computation, we also obtain

$$
\lim _{t \uparrow T} q_{t}=\underline{q} \geq q_{0}=\frac{\underline{q}^{s}\left(1-p_{0}+p_{0} \nu\right)}{\left(1-\underline{q}^{s}\right) \nu+\underline{q}^{s}\left(1-p_{0}+p_{0} \nu\right)} \Leftrightarrow B \geq p_{0},
$$

which implies that $q_{t}$ must jump up at $t=\tau$ with $p_{\tau}=c$ if $B \geq p_{0}$ (see the proof of Proposition 2). We thus have $\lim _{\lambda \rightarrow \infty} e^{-\lambda \tau}=\frac{c\left(1-p_{0}\right)}{p_{0}(1-c)}$ which is finite by assumption, and $\lim _{\lambda \rightarrow \infty} \ell=\infty$ by (10) since $\lim _{\lambda \rightarrow \infty} \underline{p}=0$. 
If $B<p_{0}$, on the other hand, $q_{t}$ may or may not jump up at $t=\tau$. If it does, the same argument as above applies. We thus focus on the case where $q_{t}$ is continuous at $t=\tau$. Now define $\bar{T}$ such that

$$
e^{-\lambda \bar{T}}=\frac{(\lambda+r) B\left(1-p_{0}\right)}{p_{0}((1-B) \lambda-B r)}
$$

which is the least amount of time that is needed for $q_{t}$ to reach $\underline{q}$ (by exerting maximum effort all the way). Note that $\lim _{\lambda \rightarrow \infty} e^{-\lambda \bar{T}}=\frac{B\left(1-p_{0}\right)}{p_{0}(1-B)}>0$. Since $q_{t}$ decreases continuously, we have $\bar{T}>\tau$ and hence that $e^{-\lambda \tau}$ must also be bounded away from zero as $\lambda \rightarrow \infty$. It then follows from (10) that $\lim _{\lambda \rightarrow 0} \ell=\infty$.

The proposition follows immediately from this lemma. When $\lambda$ tends to $\infty, q_{0}$ must be arbitrarily close to 1 . The principal is then informed almost surely, and the cost of the uninformed type waiting until $T$ diminishes to 0 . In contrast, since $\lim _{\lambda \rightarrow \infty} e^{-\lambda \tau}>0$ and $\lim _{\lambda \rightarrow \infty} \ell \rightarrow \infty$ from Lemma 8 , the agent must go through a prolonged screening phase with unobservable effort (with probability $e^{-\lambda \tau}$ ), and the benefit converges to a positive number. 